\title{
Intravenous pathogenicity of influenza virus A/H5N1/2014 isolated from pig in Ogbomoso, Nigeria
}

\author{
E. Kolawole Oladipo ${ }^{1,2, *}$, J. Kola Oloke ${ }^{2}$ and J. Adekunle Adeniji ${ }^{3}$ \\ ${ }^{I}$ Department of Microbiology, Virus Research Laboratory, Adeleke University, P.M.B. 250, Ede, Osun State, \\ Nigeria \\ ${ }^{2}$ Department of Pure and Applied Biology (Microbiology Unit), Ladoke Akintola University of Technology, P.M.B. \\ 4000, Ogbomoso, Oyo State, Nigeria \\ ${ }^{3}$ Virology Department, College of Medicine, University of Ibadan, Ibadan, Oyo State, Nigeria
}

\begin{abstract}
Understanding the pathogenicity of avian influenza viruses in poultry is an important scientific and public health challenge because of antigenic shift/drift and a source of novel, potentially human-pathogenic strains. We have previously isolated an influenza A strain (H5N1/2014/Ogbomoso) from an outbreak among pig and have now aimed to assess its pathogenicity in an avian host and to categorize it as a low or high pathogenic strain. Intravenous pathogenicity index of the isolated virus was assayed using experimental infection of 6 weeks old pathogen-specific free chicken. The peak of clinical signs was on day three post-infection, and one death was observed on day eight. The intravenous pathogenicity index of this isolate was 0.08 . This results classify this isolate as a low pathogenic avian influenza strain.
\end{abstract}

Keywords: H5N1, Influenza, Intravenous pathogenicity, Nigeria, Ogbomoso.

\section{Introduction}

Influenza viruses are single-stranded RNA viruses and a member of the Orthomyxoviridae. On the groundwork of the antigenic nature of their membranebound surface glycoproteins which are haemagglutinin (HA) and neuraminidase (NA), influenza viruses are subdivided into distinctive sub-types. Antigenic shift and drift show up regularly in the antigenic sites of HA and NA which are responsible for the mechanism of adaptation and survival of the virus. Avian influenza A viruses are referred to as low pathogenic avian influenza (LPAI) and highly pathogenic avian influenza (HPAI), based on the severity of sickness caused in poultry. Avian influenza viruses have posed a huge hazard to human and animal wellness and brought about unprecedented outbreaks among animals and humans in Asian, European and African countries (OIE, 2008; Capua and Alenxander, 2009).

Avian influenza can occur in pandemics, epidemics, localized outbreaks, and as sporadic cases. Many strains of avian influenza virus can cause varying stages of infections in poultry. LPAI can additionally cause ailment in people as reported by Alexander (2006). For example, H7N7 causes conjunctivitis, and H9N2, H7N2 and H9N2 cause influenza-like clinical infection. The continuous existence of LPAI virus in an avian populace may additionally supply possibilities for the virus to accumulate mutations and convert to a more highly pathogenic strain (Alexander, 2007).
Avian influenza viruses has been detected occasionally in many mammals of various species. These species in which avian influenza virus has been detected include cats, dogs, pigs, horses, donkeys, mink and various wild mammals (Yassine et al., 2013; CDC, 2014; Su et al., 2014; He et al., 2015). Previously confirmed low pathogenic strain may also be transformed into 'potentially pathogenic' virus by using a single mutation (CDC, 2014).

Outbreaks of avian influenza virus have occurred several time in Nigeria. This isolate of influenza virus H5N1 was obtained in 2014 from a pig population in Oyoso Sate, Nigeria, and was designated H5N1/2014/Ogbomoso. Understanding its pathogenicity is of great importance to prevent future outbreaks. Thus, this study was designed to determine the intravenous pathogenicity of this isolate.

\section{Material and Methods}

The influenza virus H5N1/2014/Ogbomoso isolate was propagated in nine to eleven days old chicken embryonated eggs. The intravenous pathogenicity index (IVPI) was carried out with accordance to the recommendation of the Office International Des Epizooties (OIE, 2008). Six weeks old specific pathogen-free chickens (SPF) with no previous history of vaccination against any subtypes of influenza virus were purchased from a poultry farm in Ogbomoso.

Ten chickens were injected intravenously into a wing vein, using $0.1 \mathrm{~mL}$ of an inoculum containing 1:10 
dilution (using sterile PBS) of harvested allantoic fluid, which was obtained from nine to eleven days old embryonated eggs having an HA titer > 4HAU. The controls were inoculated with $1 \mathrm{X}$ PBS only.

Chickens were examined daily for 10 days and scored 0 if normal, 1 if sick, 2 if very sick or paralyzed, and 3 if dead. Normally, "sick" birds would show one of the following signs and "severely sick" more than one of the following signs: general sickness, sneezing, respiratory noise, ocular nasal discharge, eye redness, swelling of head, ruffled feathers, shedding (buccal or cloacal), depression, diarrhea, cyanosis of the uncovered skins or wattles, edema of the face and head, and signs of anxiety. Dead individual chickens were rated as 3 at day of death. At the end of the 10 day observation period, the sum of the observations was totaled and divided by means of the total range of observations.

The outcomes have been interpreted as described in the WHO/OIE guide (WHO, 2002; OIE, 2008). Pathogenicity is calculated based on OIE criteria. HPAI viruses have an IVPI in 6-week-old chickens greater than 1.2 or, as an alternative, cause at least $75 \%$ mortality in 4-to 8-week-old chickens infected intravenously (WHO, 2002; OIE, 2008).

\section{Results}

Figure 1 shows the trend of the clinical signs of the infected and control birds. The control group did not show any clinical signs throughout the experimental period, while the group infected the virus shows few clinical signs such as depression, diarrhea and sneezing. The peak of the clinical signs was at day three post infection and it later drops until a death was observed on day eight. The survival rate of the birds is shown in Figure 2. On days 6 and 7 post infection there was a record of a sick bird, which subsequently died on day eight post infection.

The isolate was characterized as a low pathogenic strain of avian influenza virus in 6 weeks old SPF chickens according to the IVPI (Table 1). The average intravenous pathogenicity index of the isolate was 0.08 (Table 1). Taken together, these results classify this isolate as a LPAI virus according to WHO (2002) and OIE (2008) definition.

\section{Discussion}

Pathogenicity of influenza virus isolate is a term in assessing the virulence which can be done in chicken. The observed clinical signs during the pathogenicity test can be used to describe the virulence of such subtypes of influenza virus. Avian influenza viruses can be regarded high or low pathogenic on the basis of general IVPI and molecular classification (Lee et al., 2007). It also concerns countries worldwide as they may cause future next influenza pandemic (Pappaioanou, 2009).

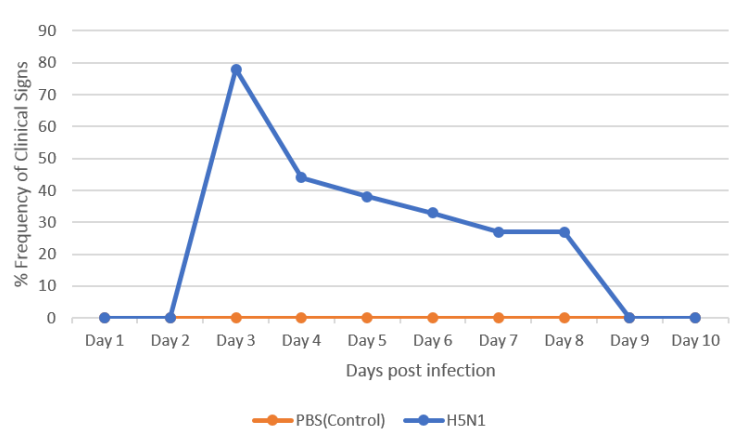

Fig. 1. Percentage trend of clinical signs with respect to days of post infection.

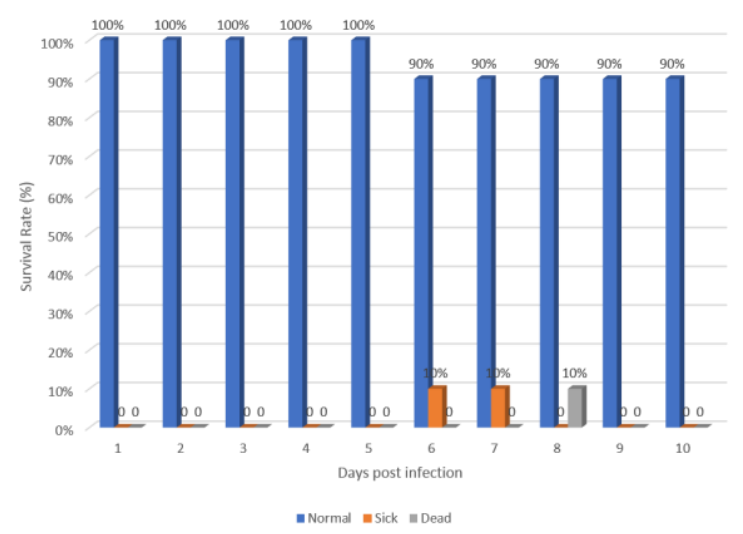

Fig. 2. Survival Rate of Birds infected intravenously with H5N1.

According to the European Union definition, this assay is an ability by means of which the level of pathogenicity of an isolate is regarded through scoring medical signs in 6 weeks SPF infected birds over a ten day period (Alexander, 2006; OIE, 2008). Hence, this isolate was categorized as a low pathogenic virus according to its IVPI. Variations in the pathogenicity and transmissibility of influenza viruses from different hosts have frequently caused problems in diagnosis, definition and the understanding of the influenza infection in poultry (Bankowaki, 1981). The mild illness observed from this study has earlier been reported that LPAI virus usually cause subclinical infections or mild illnesses in poultry (To et al., 2013; Jones et al., 2014; Pantin-Jackwood et al., 2014).

Bertran et al. (2014) has reported that infections of birds with LPAI influenza viruses have been asymptomatic while others had few clinical signs as observed from this study. Also, it has been earlier established that low pathogenic virus may also be transformed into 'potentially pathogenic virus' by way of a single factor mutation (OIE, 2008). Thus, H5N1 viruses tend to re-emerge during colder seasons in endemic areas (Normile and Enserink, 2007; Mathur et al., 2014). 
Table 1. Intravenous pathogenicity index of influenza A/H5N1/Ogbomoso/2014 isolate.

\begin{tabular}{|c|c|c|c|c|c|c|c|c|c|c|c|c|}
\hline \multirow{2}{*}{$\begin{array}{l}\text { Clinical } \\
\text { Signs }\end{array}$} & \multicolumn{10}{|c|}{ Days } & \multirow{2}{*}{$\begin{array}{l}\text { Total } \\
\text { Score }\end{array}$} & \multirow{2}{*}{$\begin{array}{c}\text { Clinical } \\
\text { Signs }\end{array}$} \\
\hline & 1 & 2 & 3 & 4 & 5 & 6 & 7 & 8 & 9 & 10 & & \\
\hline \multicolumn{13}{|c|}{ Isolate (A/H5N1/Ogbomoso/2014) } \\
\hline Normal & 10 & 10 & 10 & 10 & 10 & 9 & 9 & 9 & 9 & 9 & $95 \times 0$ & 0 \\
\hline Sick & 0 & 0 & 3 & 0 & 0 & 1 & 1 & 0 & 0 & 0 & $5 \times 1$ & 5 \\
\hline Paralyzed & 0 & 0 & 0 & 0 & 0 & 0 & 0 & 0 & 0 & 0 & $0 \times 2$ & 0 \\
\hline Dead & 0 & 0 & 0 & 0 & 0 & 0 & 0 & 1 & 0 & 0 & $1 \times 3$ & 3 \\
\hline \multicolumn{13}{|c|}{ Index $(8 / 100)=0.08$} \\
\hline \multicolumn{13}{|c|}{ Un-inoculate Control (PBS) } \\
\hline Normal & 10 & 10 & 10 & 10 & 10 & 10 & 10 & 10 & 10 & 10 & $100 \times 0$ & 0 \\
\hline Sick & 0 & 0 & 0 & 0 & 0 & 0 & 0 & 0 & 0 & 0 & $0 \times 1$ & 0 \\
\hline Paralyzed & 0 & 0 & 0 & 0 & 0 & 0 & 0 & 0 & 0 & 0 & $0 \times 2$ & 0 \\
\hline Dead & 0 & 0 & 0 & 0 & 0 & 0 & 0 & 0 & 0 & 0 & $0 \times 3$ & 0 \\
\hline Index $(0 / 10$ & $=0$ & & & & & & & & & & & \\
\hline
\end{tabular}

Herein, the pathogenicity of influenza virus A/H5N1/Ogbomoso/2014 was assessed and determined to be of low pathogenicity in 6 weeks old SPF chickens, with no previous records of vaccination against influenza virus of any subtype.

Furthermore, this finding highlighted the significance and future challenges associated with new epidemics of avian influenza virus, as this isolate was once obtained from pig which is a 'mixing vessel' in endemic and formerly infection-free countries.

\section{Acknowledgments}

The authors acknowledge the virology staff of the Prof J.K. Oloke Microbiology Research Laboratory for their assistance.

\section{Conflict of interest}

The authors declare that there is no conflict of interest.

\section{References}

Alexander, D.J. 2007. An overview of the epidemiology of avian influenza. Vaccine 25(30), 5637-5644.

Alexander, D.J. 2006. Avian influenza viruses and human health. Dev. Biol. 124, 77-84.

Bankowaki, R.A. 1981. Introduction and objectives of the symposium: Proceedings of the first symposium on avian influenza, Richmond USA, Carter Comp, Corp, pp: vii-xiv.

Bertran, K., Dolz, R. and Majo, N. 2014. Pathobiology of avian influenza virus infection in minor gallinaceous species: a review. Avian Pathol. 43(1), 9-25.

Capua, I. and Alenxander, D.J. 2009. Avian influenza and Newcastle disease: A filed and laboratory manual. Springer Verlag Italia, Milan Italy.

CDC. 2014. Centers for Disease Control and Prevention. Avian flu. Available at: http://www.cdc.gov/flu/avianflu/. Accessed on 10 February 2017.

He, S., Shi, J., Qi, X., Huang, G., Chen, H. and Lu, C. 2015. Lethal infection by a novel reassortant H5N1 avian influenza $A$ virus in a zoo housed tiger. Microbes Infect. 17(1), 54-61.

Jones, J.C., Sonnberg, S., Kocer, Z.A., Shanmuganatham, K., Seiler, P., Shu, Y., Zhu, H., Guan, Y., Peiris, M., Webby, R.J. and Webster, R.G. 2014. Possible role of songbirds and parakeets in transmission of influenza $\mathrm{A}(\mathrm{H} 7 \mathrm{~N} 9)$ virus to humans. Emerg. Infect. Dis. 20(3), 380-385.

Lee, C.W., Lee, Y.J., Swayne, D., Senne, D., Linares, D.J. and Suarez, D. 2007. Assessing potential pathogenicity of avian influenza virus: current and experimental system. Avian Dis. 51(1), 260-263.

Mathur, M.B., Patel, R.B., Gould, M., Uyeki, T.M., Bhattacharya, J., Xiano, Y., Gillaspie, Y., Chae, C. and Khazeri, N. 2014. Seasonal patterns in human A (H5N1) virus infection: analysis of global cases. PLoS One. 9(9), e106171. doi: 10.1371/journal.pone.0106171.

Normile, D. and Enserink, M. 2007. Avian influenza. With change in the seasons, bird flu returns. Science. 315(5811), 448.

OIE. 2008. OIE manual of diagnostic test and vaccine for terrestrial animal. Avian Influenza. Paris, France, pp: 465-481.

Pantin-Jackwood, M.J., Miller, P.J., Spackman, E., Swayne, D.E., Susta, L., Costa-Hurtado, M. and Suarez, D.L. 2014. Role of poultry in the spread of novel H7N9 influenza virus in China. J. Virol. 88(10), 5381-5390.

Pappaioanou, M. 2009. Highly pathogenic H5N1 avian influenza virus: Cause of the next pandemic? Comp. Immunol. Microbiol. 32, 287-300. 
Su, S., Zhou, P., Fu, X., Wang, L., Hong, M. and Lu, G. 2014. Virological and epidemiological evidence of avian influenza virus infections among feral dogs in live poultry markets, China: A threat to human health? Clin. Infect. Dis. 58(11), 1644-1646.

To, K.K., Chan, J.F., Chen, H., Li, L. and Yuen, K.Y. 2013. The emergence of influenza A H7N9 in human beings 16 years after influenza AH5N1: a tale of two cities. Lancet Infect. Dis. 13, 809-821. WHO. 2002. World Health Organization. Laboratory procedures. In WHO manual on animal influenza diagnosis and surveillance.

Yassine, H.M., Lee, C.W. and Saif, Y.M. 2013. Interspecies transmission of influenza A viruses between swine and poultry. Curr. Top. Microbiol. Immunol. 370, 227-240. 